Șerban Al. O. , Obadă B.

\title{
Locking or Non-locking Hook Plate in Treatment of Unstable Lateral Clavicle Fracture
}

Clinic of Orthopedics and Traumatology, Emergency County Hospital Constanța

\begin{abstract}
The purpose of this study was to compare the outcomes and complications of locking or nonlocking clavicular hook plate for fixation of unstable lateral clavicle fractures. All patients with unstable Neer type II lateral clavicle fractures were operated in our hospital from January 2011 to December 2012. The included participants received either locking or nonlocking clavicular hook plate operations. Demographic data, medical records and radiographs were reviewed retrospectively. At the last follow-up, shoulder function was evaluated with Constant-Murley scoring system. Our findings suggest that locking clavicular hook plates are equally useful for treating unstable lateral clavicular fractures, but in face of complicated ones, the locking hook plate would not get more benefits than nonlocking hook plate.
\end{abstract}

Keywords:

\section{Introduction}

Unstable lateral clavicle fractures (Neer type II) are usually associated with a high risk of delayed union, malunion and nonunion. Malunion can cause not only cosmetic problems, but also shortening and improper angulations, as well as subacromial impingement.

We had reported that hook plates are useful for treating unstable lateral clavicle fractures.[1]

In recent years, new device of locking clavicular hook plate was introduced and designed as anathomical precontour, limited plate contact and fixed angle construct, believed to have improvement in surgical outcome and patient satisfaction.[2], [3]

The purpose of this study was to compare the outcomes and complications of locking or nonlocking clavicular hook plate for fixation of unstable lateral clavicle fractures. 


\section{Material and methods}

All patients with unstable Neer type II lateral clavicle fractures were operated in our hospital from January 2011 to December 2012. The included participants received either locking or nonlocking clavicular hook plate operations. Figure1, figure 2

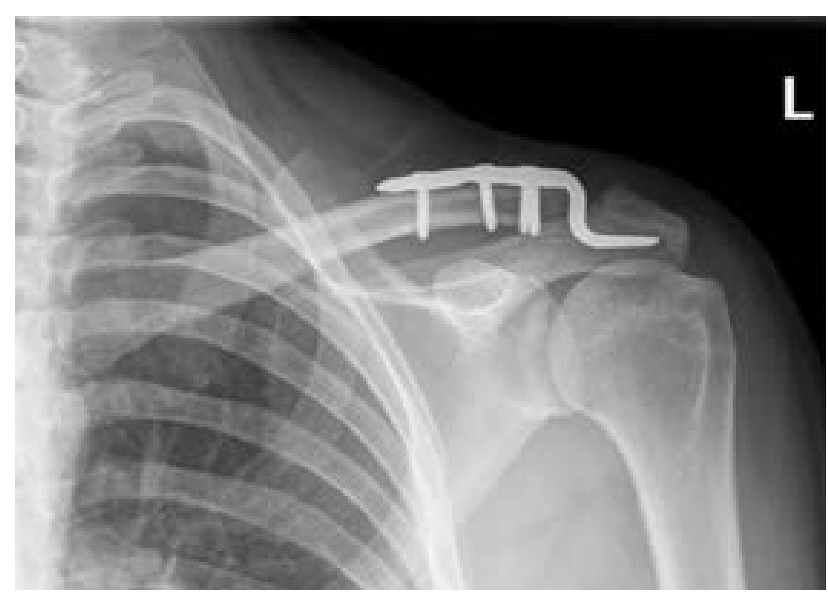

Figure 1. Locking hook plate

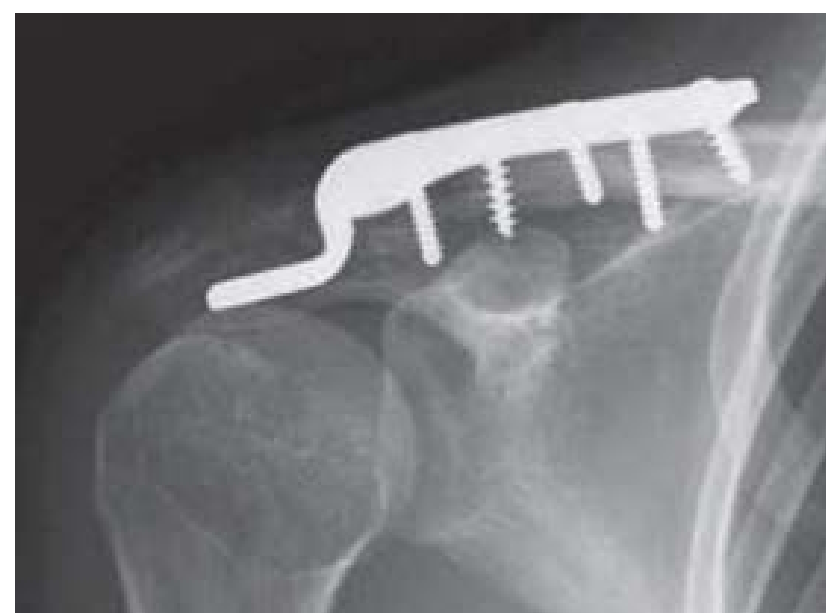

Figure 2. Nonlocking hook plate

Demographic data, medical records and radiographs were reviewed retrospectively. At the last follow-up, shoulder function was evaluated with Constant-Murley scoring system.

\section{Results}

The outcomes of 22 consecutive patients (mean age $39.40+/-14.48$ years) with unstable fractures of the lateral clavicle treated using locking clavicle hook plates were compared with those of 26 patients (mean age $38.50+/-13.34$ years) treated using a nonlocking ones.

Patients in the locking hook plate and nonlocking hook plate groups were followed up for $12.27+/-5.74$ and $21.88 .40+/-5.63$ months, respectively $(<0.001)$.

The time to hardware removal was slightly shorter in the locking hook plate group $(7+/-3.28$ months) compared with the nonlocking hook plate group (7.83+/- 3.89 months), whereas the ConstantMurley score was slightly lower in the locking hook plate group $(88.5+/-7.24)$ compared with the nonlocking group $(92.5+/-7.10)$ at final follow-up.

There are four complications in the locking hook plate group and 2 in the nonlocking hokk plate group. Figure 3, figure 4

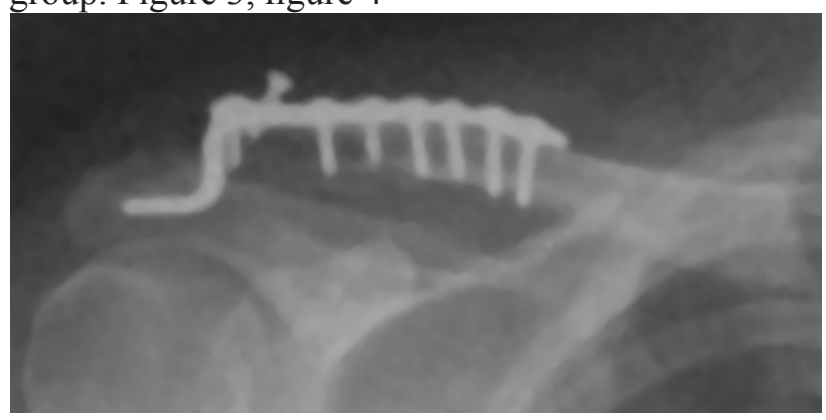

Figure 3. Screw back out in one nonlocking hook plate case

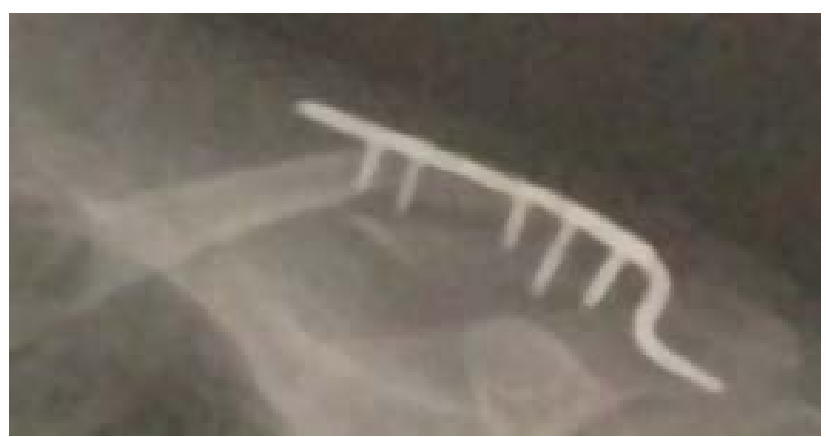

Figure 4. Loss of reduction in one locking hook plate case 
Complications in the locking hook plate group included one loss of reduction and two re-fracture and one acrimio-clavicular joint subluxation after implant removal.

Complications in the nonlocking group included on wound infection and one screw pullout. Table I

\begin{tabular}{|l|l|l|}
\hline AO Hook Plate & Non-locking & Locking \\
\hline Case no. & 26 & 22 \\
\hline Mean age (years) & $38.50+/-13.34$ & $39.40+/-14.48$ \\
\hline $\begin{array}{l}\text { Follow-up } \\
\text { (months) }\end{array}$ & $21.88 .40+/-5.63$ & $12.27+/-5.74$ \\
\hline $\begin{array}{l}\text { Time to removal } \\
\text { (months) }\end{array}$ & $7.83+/-3.89$ & $7+/-3.28$ \\
\hline $\begin{array}{l}\text { Constant-Murley } \\
\text { score }\end{array}$ & $92.5+/-7.10$ & $88.5+/-7.24$ \\
\hline Complication & $2(7.7 \%)$ & $4(18.2 \%)$ \\
\hline & $\begin{array}{l}1 \text { wound } \\
\text { infection } \\
1 \text { screws pullout }\end{array}$ & $\begin{array}{l}1 \text { loss of } \\
\text { reduction } \\
2 \text { refracture } \\
1 \text { AC joint } \\
\text { subluxation }\end{array}$ \\
\hline
\end{tabular}

\section{Conclusions}

In our study, the locking clavicular hook plate may provide an equally stable fixation with nonlocking hook plate by same removal time and same shoulder Constant-Murley score recovery.

Due to different indication in choice of implant, we find that in the locking group, the fracture pattern would be more complicated and comminuted, even more osteoporosis - this is why loss of reduction na dre-fracture happened in the group of locking hook plate.

Moreover, the design of the hook plate has its limitation. Even the precontoured locking hook plate may sometimes hardly fit the anatomy of lateral clavicle, as well as, even the longest plate could not offer the adequate fixation stability in long fracture pattern.

Our findings suggest that locking clavicular hook plates are equally useful for treating unstable lateral clavicular fractures, but in face of complicated ones, the locking hook plate would not get more benefits than nonlocking hook plate.

\section{References}

1. Wu K., Chang C.H. \& Yang R.S. (2011). Comparing hook plates and Kirschner tension band wiring for unstable lateral clavicle fractures. Orthopedics. 34(11): e718-23. DOI: 10.3928/01477447-20110922-17.

2. Klein, S.M., Badman, B.L. \& Keating CJ. (2010). Results of surgical treatment for unstable distal clavicular fractures. J. Elbow Shoulder Surg. 19(7), 1049-55. DOI: 10.1016/j.jse.2009.11.056

3. Tsuei Y.C., $\mathrm{Au}$ MK. \& Chu W. (2010). Comparison of clinical results of surgical treatment for unstable clavicular distal fractures by transacromial pins with and without tension band wire. J Chin Med Assoc. 73(12), 638-43. 